\title{
A COMMUTATIVE LOCAL RING WITH FINITE GLOBAL DIMENSION AND ZERO DIVISORS
}

\author{
BY \\ B. L. OSOFSKY(1)
}

It is well known that a commutative, local, noetherian ring (with 1) of finite global dimension must be a domain (indeed a regular local ring). For commutative local rings which are coherent or have linearly ordered ideals, finite global dimension also implies no proper zero divisors (see [6] and [7]). In this note we show that these results cannot be generalized to arbitrary commutative local rings. An example is given of a ring with global dimension 3 possessing a localization which is not a domain. This ring also has weak global dimension 2, these dimensions being the smallest possible in local rings with zero divisors. Some results on homological dimension of ideals generated by two elements in $C(R)$, the ring of all continuous real-valued functions on the reals $\boldsymbol{R}$, are also included.

1. Global dimension 3. Let $R$ be the subring of $C(R)$ consisting of piecewise sums of odd roots of polynomials and quotients thereof. That is, $f \in R$ if and only if:

(i) $f$ is a continuous function from $\boldsymbol{R}$ to $\boldsymbol{R}$ and

(ii) there exists $\left\{x_{1} \mid 1 \leqq i \leqq n\right\}$ with $-\infty=x_{1}<x_{2}<\cdots<x_{n}=+\infty$ such that $f$ restricted to $\left(x_{i}, x_{i+1}\right)$ is of the form $\sum_{i=1}^{m} p_{i}(x)^{1 / k_{i}} / \sum_{i^{\prime}=1}^{n} q_{i}(x)^{1 / j^{\prime}}$, where $p_{i}$ and $q_{i^{\prime}} \in \boldsymbol{R}[X]$ and $k_{i}$ and $j_{i^{\prime}}$ are positive odd integers.

The following properties of elements of $R$ are used to calculate its global dimension.

(a) $f \in R \Rightarrow f^{-1}[R-\{0\}]$ is a finite disjoint union of open intervals.

Proof. $\sum_{i=1}^{m} p_{i}(z)^{1 / k_{i}} / \sum_{i^{\prime}=1}^{n} q_{i^{\prime}}(z)^{1 / j_{i^{\prime}}}$ is an algebraic function, that is, it is analytic on the complex sphere except for a finite number of points $\left\{a_{k}\right\}$ where it is analytic or has a pole as a function in $\left(z-a_{k}\right)^{1 / l}\left(\right.$ or $\left.z^{-1 / l}\right)$ for some $l$. Hence it is either identically zero or has only a finite number of zeros.

(b) If $f, g \in R$ and $f \in g C(R)$, then $f \in g R$. (We will say that $f / g$ is continuous if this occurs.)

(c) For any $y \in \boldsymbol{R}$, there is a nontrivial interval $[y, u]$ (respectively $[v, y]$ ) on which $f$ is of the form $(x-y)^{n / k} p(x)$ (respectively $\left.(x-y)^{m / s} p^{\prime}(x)\right)$ where $p$ (resp. $p^{\prime}$ ) is

Presented to the Society, April 18, 1968 under the title Homological dimension in rings of continuous functions; received by the editors May 21, 1968.

( ${ }^{1}$ ) This paper was written while the author held a National Science Foundation Postdoctoral Fellowship and a Rutgers Research Council Faculty Fellowship. 
analytic in $(x-y)^{1 / k}\left(\operatorname{resp} .(x-y)^{1 / j}\right)$ at $y$, and $p(y) \neq 0$ or $p \equiv 0\left(p^{\prime}(y) \neq 0\right.$ or $\left.p^{\prime} \equiv 0\right)$. Set

$$
\begin{aligned}
o_{r} f(y) & =n / k & & \text { if } p(x) \not \equiv 0 \\
& =\infty & & \text { if } p(x) \equiv 0 \\
o_{l} f(y) & =m / j & & \text { if } p^{\prime}(x) \not \equiv 0 \\
& =\infty & & \text { if } p^{\prime}(x) \equiv 0
\end{aligned}
$$

$o_{r} f(y)\left(o_{l} f(y)\right)$ is called the right (left) order of $f$ at $y$.

(d) $\infty \neq o_{r} f(y) \leqq o_{r} g(y) \Leftrightarrow \lim _{x \rightarrow y+} g(x) / f(x)$ exists, and a symmetrical statement holds for $o_{l}$.

(e) $o_{r}(f g(y))=o_{r} f(y)+o_{r} g(y) . \quad o_{r}(f+g)(y) \geqq \min \left\{o_{r} f(y), o_{r} g(y)\right\}$, and equality holds except when $\lim _{x \rightarrow y+} f(x) / g(x)=-1$.

Symmetrical statements hold for $o_{l}$.

Let $I$ be an ideal of $R$. Set

$$
\begin{aligned}
& A=\{x \in \boldsymbol{R} \mid f(x)=0 \text { for all } f \in I\} \\
& B=\left\{x \in A \mid \text { there exists an } f \in I \text { such that } o_{r} f(x) \text { and } o_{l} f(x)<\infty,\right. \text { and for all } \\
& \qquad g \in I, g / f \text { is continuous in a neighborhood of } x\} .
\end{aligned}
$$

Clearly $A$ is closed and $B$ is contained in an open set disjoint from $A-B$, so $A-B$ is closed. Let $D=R-(A-B)$. Then $D$ is open, so $D=\bigcup\left(u_{i}, v_{i}\right)$ where $\left\{\left(u_{i}, v_{i}\right)\right\}$ is at most a countable family of disjoint open intervals.

Let $\chi_{i}$ denote the characteristic function of $\left(u_{i}, v_{i}\right)$.

If $f$ is a function defined on some closed interval $[y, z]$ which agrees with an element of $R$ there, let $f^{\#}$ denote the element of $R$ which is constant on $(-\infty, y]$ and $[z, \infty)$ and equal to $f$ on $[y, z]$.

LEMMA 1. $I=\oplus \chi_{i} I$.

Proof. Since $\left\{u_{i}\right\} \cup\left\{v_{i}\right\} \subseteq A, \chi_{i} f \in R$ for all $f \in I$. We first show that $\chi_{i} f \in I$.

If $u_{i}=-\infty$, set $f^{\prime}=f$.

If $f \equiv 0$ on $[y, z]$ where $u_{i} \in[y, z]$, set $h(x)=(x-y) /(z-y)$ on $[y, z]$. Then $f^{\prime}$ $=f h^{\#} \in I$, and $f^{\prime}$ is zero on $\left(-\infty, u_{i}\right]$ and agrees with $f$ on $\left[u_{i}, \infty\right)$.

If neither of the above cases holds, since $u_{i} \in A-B$, there is a $g \in I$ such that $g / f$ is not continuous at $u_{i}$. By (d), $f / g$ is continuous on a closed neighborhood $\left[z^{\prime}, u_{i}\right]$ or $\left[u_{i}, z\right]$. Then $f-g(f / g)^{\#}$ is zero on an interval to the left (right) of $u_{i}$ and has the same right (left) order as $f$ by (e). Multiply by a function $h^{\#}$ which equals

$$
f /\left(f-g(f / g)^{\#}\right)
$$

on the right (left) of $u_{i}$, and is linear to zero on the zero interval of $f-g(f / g)^{\#}$. Then $k=\left[f-g(f / g)^{\#}\right] h^{\#} \in I$ and $k$ (or $f-k$ ) is zero on a neighborhood to the left of $u_{i}$, and equals $f$ on some neighborhood $\left[u_{i}, z\right]$. Multiplying by an appropriate function as in the preceding case, we may obtain a function $f^{\prime} \in I$ which agrees with $f$ on $\left[u_{i}, z\right]$ and is zero on $\left(-\infty, u_{i}\right]$. 
Similarly, we may find an $f^{\prime \prime} \in I$ which is zero on $\left[v_{i}, \infty\right)$ and agrees with $f$ on some $\left[y, v_{i}\right]$ where $y>z$.

Let $[a, b] \subset\left(u_{i}, z\right],[c, d] \subset\left[y, v_{i}\right)$ be nontrivial intervals where $f$ is either never zero or identically zero. Let $g^{\#}=1$ on $(-\infty, a], 0$ at $b$, and linear on $[a, b]$. Let $h^{\#}$ be 1 on $[d, \infty), 0$ at $c$, and linear on $[c, d]$. Set $k=\chi_{i} f-f^{\prime} g^{\#}-f^{\prime \prime} h^{\#}$. Then $k \equiv 0$ on $(-\infty, a]$ $\cup[d, \infty)$. Let $m^{\#} \equiv 1$ on $[b, c]$ and $k / f$ (or linear to zero if $f \equiv 0$ ) on $[a, b]$ and $[c, d]$. Then $k-f m^{\#} \equiv 0$, so $\chi_{i} f=f^{\prime} g^{\#}+f^{\prime \prime} h^{\#}+f m^{\#} \in I$.

By (a), there are only a finite number of $i$ such that $\chi_{i} f \neq 0$ for $f \in I$. Hence $I=\sum \chi_{i} I$. Since $\left\{\left(u_{i}, v_{i}\right)\right\}$ are disjoint, the sum is direct.

To calculate the homological dimension of $I$, hd $(I)$, we need only calculate that of each $\chi_{i} I$. Hence we will assume that $I=\chi_{i} I$, that is, $A \supseteq(-\infty, u] \cup[v, \infty)$ and $A \cap(u, v)=B$.

For each $x \in B$, let $N_{x}$ be an open interval containing $x$ and $f_{x}$ a function in $I$ such that $g / f_{x}$ is continuous on $N_{x}$ for all $g \in I$. Since $f_{x}$ has an isolated zero at $x$, the same $N_{x}$ will work for all $g$.

For each $y \in(u, v)-B$, let $f_{y} \in I$ and $N_{y}$ an open interval containing $y$ such that $f_{y} \neq 0$ on $N_{y}$. This can be done since $y \notin A$.

There are three situations to consider at the endpoints $u$ and $v$.

(L1) There exists $f_{u} \in I$ of minimal noninfinite order $o_{r} f(u)$, or $u=-\infty$ and $f_{u}$ is nonzero in a neighborhood $(-\infty, w)$.

(L2) $\infty \neq \inf _{f \in I}\left\{o_{r} f(u)\right\}=\alpha$, and no $f \in I$ has $o_{r} f(u)=\alpha$.

(L3) $o_{r} f(u)=\infty$ for all $f \in I$.

(R1), (R2), and (R3) are the symmetrical statements for $v$ and $o_{l} f(v)$.

LEMMA 2.' Either $I$ is cyclic or $I=\bigcup_{i=0}^{\infty} g_{i} R$, where $\left(g_{i} / g_{i+1}\right)^{\#}$ is continuous on $[u, v]$ and equal to zero at at least one endpoint.

Proof. Case (i). Assume (L1) and (R1) hold. Let $N_{u}\left(N_{v}\right)$ be half open intervals with left endpoint $u$ (right endpoint $v$ ) such that $f_{u} \neq 0$ on $N_{u}-\{u\}\left(f_{v} \neq 0\right.$ on $\left.N_{v}-\{v\}\right)$ and $f \mid f_{u}\left(f \mid f_{v}\right)$ is continuous for all $f \in I .\left\{N_{z} \mid z \in[u, v]\right\}$ is an open cover of the compact set $[u, v]$. Hence one may extract a finite subcover, say

$$
\left\{N_{z(i)} \mid 1 \leqq i \leqq m, z(i)<z(i+1)\right\} \text {. }
$$

By using smaller or fewer intervals if necessary, one may assume $z(i) \notin N_{z(j)}$ if $i \neq j$. Let $\left(a_{i}, b_{i}\right)$ be a subinterval of $N_{z(i)} \cap N_{z(i+1)}$. Let $h_{i}^{\#}$ be 1 on $\left[b_{i-1}, a_{i}\right]$ and linear to zero on $\left[a_{i-1}, b_{i-1}\right]$ and $\left[a_{i}, b_{i}\right]$. (The modifications at $i=1$ and $m$ are obvious.) Let $f=\sum_{i=1}^{m}(-1)^{v(i)} f_{z(i)} h_{i}^{\#}$, where $v(i)$ is selected so that

$$
(-1)^{v(i)+v(i+1)} f_{z(i)} f_{z(i+1)} \geqq 0
$$

on $(z(i), z(i+1))$. Then $f \in I$ and $f^{-1}[0]=A$. Moreover, for all $g \in I, g / f$ is continuous at each zero of $f$, so $g=f(g / f)^{\#} \in f R$ and $I$ is cyclic.

Case (ii). If (R1) or (L1) holds, define $f_{u}^{(k)}$ and $N_{u}^{(k)}$ or $f_{v}^{(k)}$ and $N_{v}^{(k)}$ as in Case (i). If (L3) ((R3)) holds, let $\left\{w_{i}\right\}$ be a sequence decreasing monotonically to $u\left(\left\{w_{i}^{\prime}\right\}\right.$ 
increasing monotonically to $v)$. Let $N_{u}^{(k)}=\left[u, w_{k}\right), f_{u}^{(k)} \equiv 0\left(N_{v}^{(k)}=\left(w_{k}^{\prime}, v\right], f_{v}^{(k)} \equiv 0\right)$. If (L2) ((R2)) holds, let $f_{u}^{(k)}$ have right order $\alpha+1 /(2 k+1)$ at $u\left(o_{l} f_{v}^{(k)}(v)=\alpha^{\prime}+\right.$ $1 /(2 k+1))$ and let $f_{u}^{(k)} \neq 0$ on $N_{u}^{(k)}-\{u\}\left(f_{v}^{(k)} \neq 0\right.$ on $\left.N_{v}^{(k)}-\{v\}\right)$. Patch up $f^{(k)}$ from these intervals exactly as $f$ was obtained in Case (i), making sure the patching intervals $\left(a_{1}, b_{1}\right)$ and $\left(a_{m-1}, b_{m-1}\right)$ lie between $w_{k+1}$ and $w_{k}$ and between $w_{k}^{\prime}$ and $w_{k+1}^{\prime}$ respectively if (L3) or (R3) holds.

Let $g \in \dot{I}$. In case (L2) or (R2) holds, some $f^{(k)}$ has $o_{r} f^{(k)}(u)<o_{r} g(u)$. and/or $f^{(j)}$ has $o_{l} f^{(j)}(v)<o_{l} g(v)$. In case (L3) or (R3), $g \equiv 0$ on $\left[u, w_{k}\right]$ or $\left[w_{j}^{\prime}, v\right]$. In any case, taking $m=$ the larger of $j$ and $k$, we get $g \in f^{(m)} R$. Hence $I=\bigcup_{k=0}^{\infty} f^{(k)} R$, and $\left(f^{(k)} \mid f^{(k+1)}\right)^{\#}$ is defined and equal to zero at $u$ if (L2) or (L3) holds or at $v$ if (R2) or (R3) holds.

LEMMA 3. Let $J$ be an ideal of the form $\bigcup_{i=0}^{\infty} g_{i} R$, where there exists a continuous function $g_{i} / g_{i+1}$ which is equal to zero on any interval where $g_{i} i s$. Then J has dimension $\leqq 1$.

Proof. Let $F$ be a free $R$-module with basis $\left\{b_{k} \mid k \in \omega\right\}$, and let $\phi: F \rightarrow J$, $\phi\left(b_{k}\right)=g_{k}$. Clearly

$$
\operatorname{ker}(\phi) \supseteq \sum_{j<k}\left(b_{j}-\frac{b_{k} g_{j}}{g_{k}}\right) R=K .
$$

Let $\phi(r)=0$, where $r=\sum_{k=0}^{m} b_{k} f_{k}$. Set $s=\sum_{k=0}^{m}\left(b_{k}-b_{m+1} g_{k} / g_{m+1}\right) f_{k}$. Then $s \in K$ and $r-s=b_{m+1}\left(\sum_{k=0}^{m} f_{k} g_{k} / g_{m+1}\right) \in \operatorname{ker}(\phi)$. Hence $\left(\sum_{k=0}^{m} f_{k} g_{k} / g_{m+1}\right) g_{m+1}=0$. Since $\sum_{k=0}^{m} f_{k} g_{k} / g_{m+1}$ is zero whenever $g_{m+1}$ is by hypothesis, $\sum_{k=0}^{m} f_{k} g_{k} / g_{m+1} \equiv 0$ and $K=\operatorname{ker}(\phi)$. Since

$$
\left(b_{j}-\frac{b_{k} g_{j}}{\dot{g}_{k}}\right)=\sum_{n=j}^{k-1}\left(b_{h}-\frac{b_{h+1} g_{h}}{g_{h+1}}\right) \frac{g_{j}}{g_{h}},
$$

$K=\sum_{k=0}^{\infty}\left(b_{k}-b_{k+1} g_{k} / g_{k+1}\right) R$. Moreover, in any sum $\sum_{k=j}^{m}\left(b_{k}-b_{k+1} g_{k} / g_{k+1}\right) f_{k}$ where $f_{j} \neq 0$, one basis element of $F$, namely $b_{j}$, occurs with nonzero coefficient. Hence $\left\{b_{k}-b_{k+1} g_{k} / g_{k+1} \mid k \in \omega\right\}$ is a free basis for $\operatorname{ker}(\phi)$.

We observe that $J$ has weak dimension 0 , since

$$
F=\sum_{k=0}^{n}\left(b_{k}-\frac{b_{k+1} g_{k}}{g_{k+1}}\right) R \oplus \sum_{k=n+1}^{\infty} b_{k} R
$$

implies any finitely generated submodule of $\operatorname{ker}(\phi)$ is left fixed by some map from $F$ to $\operatorname{ker}(\phi)$.

\section{THEOREM 1. $R$ has global dimension 3.}

Proof. By the Global Dimension Theorem of [1], it is sufficient to show that every ideal $I$ has dimension $\leqq 2$, and at least one has dimension 2 .

If $I$ is cyclic, say $I=f R$, let

$$
0 \rightarrow(0: f) \rightarrow R \rightarrow f R \rightarrow 0
$$


be exact, where $R \rightarrow f R$ takes 1 to $f$. Clearly every $\chi_{i}(0: f)$ satisfies (L2) or (R2) at all finite endpoints, so by Lemma $2, \chi_{i}(0: f)$ satisfies the hypotheses of Lemma 3. Hence $f R$ has dimension at most 2. Moreover, if $o_{r} f(x)=\infty \neq o_{l} f(x)$ then in the localization of $R$ at $x,(0: f)$ is a nonzero module annihilated by $f \neq 0$ which is in the maximal ideal of the localization. Since projective modules over a local ring are free, the localization of $(0: f)$ is not projective over the localization of $R$. Hence $(0: f)$ is not $R$-projective, and hd $(f R)=2$.

Lemma 3 also gives the required result when each $\chi_{i} I$ satisfies (L2) or (L3) and (R2) or (R3) at the appropriate endpoint.

We are left with the case where $\chi_{1} I$ satisfies (L1) or (R1) at a finite endpoint, but not both. As in Lemma 2, $\chi_{i} I$ is a countable union of $f^{(k)} R$, where $\left(f^{(k)} / f^{(k+1)}\right)^{\#}$ is a nonzero constant on one of $(-\infty, u]$ or $[v, \infty)$, say the former. Define $F, \phi,\left\{b_{k}\right\}$ and $K$ as in Lemma 3. Since

$$
\left(f^{(h)} \mid f^{(n+1)}\right)^{\#}\left(f^{(j)} \mid f^{(h)}\right)^{\#}=\left(f^{(j)} \mid f^{(h+1)}\right)^{\#}
$$

is still valid in this case (the indicated functions are completely determined by their values on $(u, v)$ ),

$$
K=\oplus\left(b_{k}-b_{k+1}\left(f^{(k)} / f^{(k+1)}\right)^{\#}\right) R
$$

follows exactly as in Lemma 3, but it is no longer true that $K=\operatorname{ker}(\phi)$. Indeed, for $r \in \operatorname{Ker}(\phi)$, all we can conclude from the proof of Lemma 3 is that there exists an $s \in K$ with $r-s=b_{m+1} g$ for some $g$ where $g \equiv 0$ on $[u, \infty)$. Then

$$
\left(b_{0}-b_{m+1}\left(f^{(0)} / f^{(m+1)}\right)^{\#}\right) g=b_{0} g-b_{m+1} c g
$$

where $c$ is a nonzero constant, so $b_{m+1} g \in K+b_{0} J$, where $J$ is the ideal of functions of $R$ which vanish on $[u, \infty)$. Hence $\operatorname{ker}(\phi)=K+b_{0} J$. Since no nonzero element of $J$ annihilates any $\left(f^{(k)} \mid f^{(k+1)}\right)^{\#}$, one checks that $K \cap b_{0} J=0$ so the sum is direct. Since $K$ is projective and $J$ is (R2) at its only boundary point of $A$, hd $(J)=1$. Thus $\chi_{i} I$ also has dimension $=2$ in this case.

We note that any localization of $R$ at the ideal of all functions vanishing at a given point will be a commutative local ring with global dimension $=3$ and zero divisors. Moreover, by the observation at the end of Lemma 3, any ideal of $R$ has weak dimension at most 1 , and since $f R_{M}$ cannot be flat if $f$ is a zero divisor in the local ring $R_{M}$, any localization of $R$ at a point will have weak global dimension $=2$.

2. The full ring of continuous functions $C(R)$. Lemma 3 looks very much like the first step in the projective resolution of [5]. Indeed, it is.

Let $f, g \in C(\boldsymbol{R})$. We say $f>g$ if there exists a function $f^{-1} g \in C(\boldsymbol{R})$ such that $f\left(f^{-1} g\right)=g$ and $f^{-1} g=0$ whenever $g=0$. An ideal $I$ is called directed if for all $f, g \in I$ there is an $h \in I$ with $h>f$ and $h>g$. In Lemma 3, the proof that $K=\operatorname{ker}(\phi)$ clearly also shows that $\mathscr{P}_{I^{\prime}}$ is exact at $P_{0}\left(I^{\prime}\right)$ in $[5,3.1]$, where $I^{\prime}$ is any set of generators for a directed ideal. Since $f>g>h \Rightarrow\left(f^{-1} g\right)\left(g^{-1} h\right)=f^{-1} h$, the remainder of 
the proof in [5] goes through to show that $\mathscr{P}_{1}$, is a projective resolution of $I$. Hence we may use the results of [5] for directed ideals of $C(\boldsymbol{R})$.

Let $X \subseteq \boldsymbol{R}$. Let

$$
\begin{aligned}
& M_{X}=\{f \in C(R) \mid f(x)=0 \text { for all } f \in X\}, \\
& M_{X}^{\prime}=\left\{f \in M_{X} \mid f(x)=0 \Rightarrow x \in \text { closure of } X\right\} .
\end{aligned}
$$

$M_{X}^{\prime}$ generates $M_{X}$, since $d(x)=$ distance of $x$ from the closure of $X$ is a well-defined element of $M_{X}^{\prime}$, and $f \in M_{X} \Rightarrow f \in\left(d+|f|^{1 / 2}\right) C(R)$ where $d+|f|^{1 / 2} \in M_{X}^{\prime}$.

Proposition 1. If $\varnothing \neq X$ is not dense, then hd $\left(M_{X}\right) \geqq 2$.

Proof. Let $\left\{g_{i} \mid i \in \omega\right\} \subseteq M_{X}^{\prime}$. Set $m_{i}(x)=\max \left\{g_{i}(x), 1\right\}$. Then

$$
h(x)=\left[\sum_{i=0}^{\infty} \frac{1}{i^{2} m_{i}(x)}\left|g_{i}(x)\right|\right]^{1 / 2} \in M_{X}^{\prime}
$$

and $h>g_{i}$ for all $i$. By 7.1 of [5], if hd $\left(M_{X}\right) \leqq 1$, there is a directed ideal $I$ generated by a countable subset of $M_{X}^{\prime}$ with hd $(I)=0$.

Let $x$ belong to the boundary of the closure of $X$. Localize $C(\boldsymbol{R})$ at $x$. Then $I$ is still a strictly ascending union of cyclic modules over the localization. But any element in a free basis for $I$ cannot be properly contained in another cyclic module since it is not divisible by an element of the radical. Hence the localization of $I$ is not projective. (See $[4$, Lemma 6].)

We thus conclude hd $\left(M_{X}\right) \geqq 2$.

CoRollary 1. Let $f$ be a zero divisor of $C(\boldsymbol{R})$. Then hd $(f C(\boldsymbol{R})) \geqq 3$.

Proof. $(0: f)=M_{X}$, where $X=\{x \in \boldsymbol{R} \mid f(x) \neq 0\}$ and $0 \rightarrow(0: f) \rightarrow C(\boldsymbol{R}) \rightarrow f C(\boldsymbol{R})$ $\rightarrow 0$ is exact. Moreover, $X$ is not empty and not dense.

COROLlaRY 2. The continuum hypothesis implies hd $\left(M_{X}\right)=2$ if $\varnothing \neq X$ is not dense, and $\mathrm{hd}(f C(\boldsymbol{R}))=3$ for any zero divisor $f$.

Proof. Since elements of $C(\boldsymbol{R})$ are determined by their values on the rationals, $C(R)$ has cardinality $\left(2 \aleph_{0}\right)^{\aleph_{0}}$. Hence the continuum hypothesis implies $M_{X}$ is $\boldsymbol{\aleph}_{1}$-generated, so by [5, A.2] hd $\left(M_{X}\right) \leqq 2$. Since $(0: f)$ is an $M_{X}$, hd $(f C(R)) \leqq 3$.

In the ring $R$ of $\S 1$, it is easy to see that any finitely generated ideal is a direct sum of cyclics, since (L2), (L3), (R2) and (R3) require an ideal to be infinitely generated at a point. There is no such nice decomposition of finitely generated ideals of $C(R)$. (For example, look at the ideal generated by $x \sin 1 / x$ and $x \cos 1 / x$.) However, the continuum hypothesis can be used to get an upper bound on the dimension of an ideal generated by two elements.

Proposition 2. Let $f, g \in C(\boldsymbol{R})$. Then the continuum hypothesis implies

$$
\text { hd }(f C(\boldsymbol{R})+g C(\boldsymbol{R})) \leqq 4 .
$$


Proof. We have an exact sequence

$$
0 \rightarrow g C(\boldsymbol{R}) \rightarrow f C(\boldsymbol{R})+g C(\boldsymbol{R}) \rightarrow f C(\boldsymbol{R}) / f C(\boldsymbol{R}) \cap g C(\boldsymbol{R}) \rightarrow 0 .
$$

By Corollary 2, hd $(g C(\boldsymbol{R}))$ and hd $(f C(\boldsymbol{R}))$ are either 0 or 3. Hence if

$$
\text { hd }(f C(\boldsymbol{R}) \cap g C(\boldsymbol{R})) \leqq 3,
$$

the result will follow by Theorem 1.2 of [2].

Let

$$
A=\{x \in R \mid \text { there exists a neighborhood } N \text { of } x \text { such that either } f / g \text { or } g / f
$$

is continuous on $N\}$.

Then $A$ is open, say $A=\bigcup_{i}\left(u_{i}, v_{i}\right)$ where $\left\{\left(u_{i}, v_{i}\right)\right\}$ are disjoint open intervals and $f(x)=g(x)=0$ for all $x \in R-A$. Let $x \in A, f(x) \neq 0, g(x) \neq 0$. Let $(-1)^{j}=$ $f(x) g(x) /|f(x) g(x)|$. Let $N_{x}$ be a maximal open interval containing $x$ on which $f / g$ does not change sign. Let

$$
\begin{aligned}
k^{\prime}(y) & =f(y), & & |f(y)| \leqq|g(y)|, y \in N_{x}, \\
& =(-1)^{j} g(y), & & |g(y)|<|f(y)|, y \in N_{x} .
\end{aligned}
$$

The $N_{x}$ are either disjoint, coincide, or intersect on an interval where $f \equiv 0 \equiv g$, so $k^{\prime}$ is a well-defined function continuous on each $N_{x}$.

Select some $N_{x}=I_{0}$, and let $k=k^{\prime}$ on $I_{0}$. Assume the open interval $I_{\beta}$ and a function $k_{\beta}$ on $I_{\beta}$ defined for all $\beta<\alpha$ such that $I_{\beta} \subseteq I_{\gamma}$ and $k_{y}$ extends $k_{\beta}$ for all $\beta<\gamma ; N_{y} \cap I_{\beta} \neq \varnothing \Rightarrow N_{y} \subseteq I_{\beta} \Rightarrow k= \pm k^{\prime}$ on $N_{y}$; and if $k=f$ (or $g$ ) on some portion of $N_{y} \subseteq I_{\beta}$ and $-f$ (or $-g$ ) on $N_{x} \subseteq I_{\beta}$, then $f / g$ (or $g / f$ ) $=0$ somewhere between $N_{x}$ and $N_{y}$. If $\alpha$ is a limit ordinal, $I_{\alpha}=\bigcup_{\beta<\alpha} I_{\beta}$ has the required properties. If $\alpha=\beta+1$, let $I_{\beta}=(a, b)$. If $a \neq u_{i}$ (or $b \neq v_{i}$ ), then $f / g$ or $g / f=0$ at $a$ (or $b$ ), say the former. Since $f / g$ is continuous at $a$ (or $b$ ), $|g| f \mid>1$ in some neighborhood $M_{a}$ of $a$. Intervals where $f \equiv 0 \equiv g$ cause no problem, as each $N_{y} \subseteq\left(u_{i}, v_{i}\right)$ contains a point where $f \neq 0$ unless $f \equiv 0 \equiv g$ on $\left(u_{i}, v_{i}\right)$. Hence any $N_{y} \subseteq I_{\beta} \cap M_{a}$ has $k=f$ or $k=-f$ on $N_{y}$. For any $N_{y}$ with $N_{y} \cap M_{a} \neq \varnothing$, let $k=(-1)^{j} k^{\prime}$, where $j$ is selected so that $k=f$ or $k=-f$ on $M_{a}$. The construction for $g / f(a)=0$ or $b \neq v_{i}$ is symmetrical. Let $I_{\alpha}=$ the smallest open interval containing $I_{\beta}$ and all $N_{y}$ with $N_{y} \cap M_{b} \neq \varnothing$ or $N_{y} \cap M_{a} \neq \varnothing$, and let $k(x)=0$ for all $x \in I_{\beta}, x \notin N_{y}$ for any $y \in I_{\beta}$. By this procedure we eventually get an $I_{a}=\left(u_{i}, v_{i}\right)$ (indeed for some countable ordinal $\alpha$ ), and $k$ defined on $\left(u_{i}, v_{i}\right)$. Do this for each $i$, and let $k=0$ on $R-A$.

Let

$$
\begin{aligned}
X_{f} & =\{x \in \boldsymbol{R} \mid \text { no } s \in C(\boldsymbol{R}) \text { has } k=f s \text { in a neighborhood of } x\}, \\
X_{g} & =\{x \in \boldsymbol{R} \mid \text { no } s \in C(\boldsymbol{R}) \text { has } k=g s \text { in a neighborhood of } x\}, \\
X & =X_{f} \cup X_{g} .
\end{aligned}
$$

We will show $f C(\boldsymbol{R}) \cap g C(\boldsymbol{R})=k M_{\mathbf{X}}$. 
Let $m \in M_{X}, x \in \boldsymbol{R}$. Since $f, g$ and $f / g, g / f$ are all continuous whenever denominators are nonzero, $k / f$ and $k / g$ are bounded in a neighborhood of $x$. If one of them is not (or cannot be made) continuous at $x$, then $x \in X$, so $m$ is zero at $x$ and

$$
\begin{aligned}
\lim _{y \rightarrow x} m(k / f)(y) & =\lim _{y \rightarrow x} m(k / g)(y)=0 \\
& =\text { the value of the corresponding function at } x .
\end{aligned}
$$

If $k / f$ and $k / g$ are continuous at $x$, so too is $m k / f$ and $m k / g$. Hence

$$
k M_{X} \subseteq f C(\boldsymbol{R}) \cap g C(\boldsymbol{R}) .
$$

Let $f r=g s, r, s \in C(\boldsymbol{R})$. Let $D=\{x \in \boldsymbol{R} \mid k(x) \neq 0\}$. Then

$$
\begin{aligned}
(f r \mid k)(x) & = \pm r(x) & & x \in D \text { and }|f(x)| \leqq|g(x)|, \\
& = \pm s(x) & & x \in D \text { and }|f(x)|>|g(x)| .
\end{aligned}
$$

Let $b \in$ the closure of $D$. Assume $r(b) \neq 0$, say $r(b)>0$. Then there exists a neighborhood of $N$ of $b$ on which $r>0$. Let $\left\{a_{i} \mid i \in \omega\right\} \subseteq D \cap N$ be any sequence approaching $b$. Then $\lim _{i \rightarrow \infty} f\left(a_{i}\right) / g\left(a_{i}\right)=s(b) / r(b)$. If $|s(b) / r(b)|>1$, then $|f / g|>1$ on some neighborhood $M$ of $b$ with $M \subseteq N$. Then $(f r / k)\left(a_{i}\right)=s\left(a_{i}\right)$ (or $-s\left(a_{i}\right)$ ) on $M \cap\left\{a_{i}\right\}$, so $\lim _{i \rightarrow \infty}(f r / k)\left(a_{i}\right)=s(b)$ (or $\left.-s(b)\right)$. If $|s(b) / r(b)|<1, \lim _{i \rightarrow \infty}(f r / k)\left(a_{i}\right)= \pm r(b)$. If $r(b)=s(b)($ or $-s(b))$, then $b \in N_{b}$ and $\lim _{a_{i} \rightarrow \infty} f / k\left(a_{i}\right)=1$ or -1 , so

$$
\lim _{a_{i} \rightarrow \infty} f r / k\left(a_{i}\right)= \pm r(b) \text {. }
$$

Hence if $r(b) \neq 0, \lim _{i \rightarrow \infty}(f r \mid k)\left(a_{i}\right)$ depends only on $b$, and not on $\left\{a_{i} \mid i \in \omega\right\}$. By symmetry, if $s(b) \neq 0, \lim _{i \rightarrow \infty}(f r \mid k)\left(a_{i}\right)$ is independent of $\left\{a_{i} \mid i \in \omega\right\}$. Clearly if $r(b)=s(b)=0, \lim _{i \rightarrow \infty}(f r / k)\left(a_{i}\right)=0$. Hence $f r / k$ may be extended to a continuous function on the closure of $D$. This in turn may be extended to a function $h$ in $C(R)$. Moreover, since $h k=f r=g s, h(x)$ must be zero whenever $x \in X$. Hence $h \in M_{X}$, so $f r \in k M_{x}$.

Since $0 \rightarrow M_{A} \rightarrow M_{X} \rightarrow k M_{X} \rightarrow 0$ is exact and $M_{A}$ and $M_{X}$ have dimension at most $2, k M_{X}=f C(\boldsymbol{R}) \cap g(C(R))$ has dimension at most 3. Hence

$$
\text { hd }(f C(\boldsymbol{R})+g C(\boldsymbol{R})) \leqq 4 .
$$

We note that if $X \supseteq$ the boundary of the interior of $R-A$, then $M_{A}$ is a direct summand of $M_{X}$ and $k M_{X}$ has dimension 2, so

$$
\text { hd }\left(f C(\boldsymbol{R}) / k M_{X}\right)=3=\text { hd }(f C(\boldsymbol{R})+g C(\boldsymbol{R})) \text {. }
$$

For example, hd $([x \sin (1 / x)] C(\boldsymbol{R})+[x \cos (1 / x)] C(\boldsymbol{R}))=3$.

However, $k M_{x}$ may have dimension 3 , in which case no further information is obtainable about hd $(f C(\boldsymbol{R})+g C(\boldsymbol{R}))$ by this method. For example, let

$$
\begin{array}{ll}
f(x)=x, g(x)=0 & x \in(-\infty, 0], \\
f(x)=x \sin (1 / x), g(x)=x & x \in[0,1 / 2], \\
f(x)=(1-x) \sin 2, g(x)=1-x & x \in[1 / 2,1], \\
f(x)=0, g(x)=0 & x \in[1, \infty) .
\end{array}
$$


Then $X=\{0\}$, and $k M_{X}$ corresponds to the (R1) but not (L1) case of Theorem 1 . If we take a set of generators of $k M_{X}$, say $\left\{f_{\alpha}\right\}$, with an element $f_{0} C(\boldsymbol{R}) \subseteq f_{\alpha} C(\boldsymbol{R})$ for all $\alpha$, precisely as in Theorem 1 we get a free module $F=\oplus b_{\alpha} C(R)$ and an epic $\phi: F \rightarrow k M_{X}$ such that

$$
\operatorname{ker}(\phi)=\sum\left(b_{\alpha}-b_{\beta}\left(f_{\alpha} / f_{\beta}\right)^{\#}\right) C(\boldsymbol{R}) \oplus b_{0} M_{(-\infty, 1]} .
$$

Then hd $(\operatorname{ker}(\phi)) \geqq$ hd $\left(M_{(-\infty, 1]}\right) \geqq 2$, so hd $\left(k M_{X}\right) \geqq 3$.

Finally, we note that if the results of this section could be generalized to any finitely generated ideal of $C(\boldsymbol{R})$, that is, if there were a $k \in \omega$ such that

$$
\text { hd }\left(\sum_{i=1}^{m} f_{i} C(\boldsymbol{R})\right) \leqq k
$$

for any finite set $\left\{f_{i}\right\}$ of elements of $C(R)$, then by Proposition 3 of [1], any $\boldsymbol{N}_{1}$ generated ideal would have dimension at most $k+2$, and the continuum hypothesis would imply $C(\dot{\boldsymbol{R}})$ has finite global dimension.

\section{BIBLIOGRAPHY}

1. M. Auslander, On the dimension of modules and algebras. III, Nagoya Math. J. 9 (1955), 67-77.

2. I. Kaplansky, Projective modules, Ann. of Math. 68 (1958), 372-377.

3. - Homological dimension of rings and modules, Mimeographed notes, University of Chicago, Chicago, Ill., 1959.

4. B. Osofsky, Global dimension of valuation rings, Trans. Amer. Math. Soc. 126 (1967), 136-149.

5. - Homological dimension and the continuum hypothesis, Trans. Amer. Math. Soc. 132 (1968), 217-230.

6. - Global dimension of commutative rings with linearly ordered ideals, J. London Math. Soc. 44 (1969), 183-185.

7. L. Small, Some consequences of finite projective dimension, Report to the meeting on Rings and Modules at the Mathematisches Forschungsinstitut, Oberwolfach, Germany, 1968.

INSTITUTE FOR ADVANCED STUDY,

Princeton, New Jersey

Rutgers, The State University,

New BRUnSWick, NeW JeRSEY 